Bull. Korean Math. Soc. 48 (2011), No. 4, pp. 713-724

DOI 10.4134/BKMS.2011.48.4.713

\title{
DYNAMICS OF TRANSCENDENTAL ENTIRE FUNCTIONS WITH SIEGEL DISKS AND ITS APPLICATIONS
}

\author{
KoH Katagata
}

\begin{abstract}
We study the dynamics of transcendental entire functions with Siegel disks whose singular values are just two points. One of the two singular values is not only a superattracting fixed point with multiplicity more than two but also an asymptotic value. Another one is a critical value with free dynamics under iterations. We prove that if the multiplicity of the superattracting fixed point is large enough, then the restriction of the transcendental entire function near the Siegel point is a quadraticlike map. Therefore the Siegel disk and its boundary correspond to those of some quadratic polynomial at the level of quasiconformality. As its applications, the logarithmic lift of the above transcendental entire function has a wandering domain whose shape looks like a Siegel disk of a quadratic polynomial.
\end{abstract}

\section{Introduction}

Let $f: \mathbb{C} \rightarrow \mathbb{C}$ be an entire function. The Fatou set $F(f)$ is the set of normality in the sense of Montel for the family $\left\{f^{n}\right\}_{n=1}^{\infty}$, where $f^{n}=f \circ \cdots \circ f$ is $n$ iterates of $f$. The Julia set $J(f)$ is the complement $\mathbb{C} \backslash F(f)$. Let $z_{0}$ be a fixed point of $f$ with $f^{\prime}\left(z_{0}\right)=e^{2 \pi i \alpha}$, where $0<\alpha<1$ is irrational. The entire function $f$ is locally linearizable at the fixed point $z_{0}$ if there exists a conformal map $\varphi$ near $z_{0}$ with $\varphi\left(z_{0}\right)=0$ such that $\varphi \circ f \circ \varphi^{-1}(z)=R_{\alpha}(z)=e^{2 \pi i \alpha} z$. In this case, we call the irrational number $\alpha$ the rotation number. The entire function $f$ is locally linearizable at $z_{0}$ if and only if $z_{0}$ belongs to the Fatou set. The Fatou component $\Delta$ containing $z_{0}$ is called the Siegel disk centered at $z_{0}$. The Siegel disk $\Delta$ is the largest domain on which $f$ is conformally conjugate to the rotation $R_{\alpha}$. For the irrational number $\alpha$, we consider the continued fraction expansion $\alpha=\left[a_{1}, a_{2}, \ldots, a_{n}, \ldots\right]$, where each $a_{n}$ is a positive integer. Then the sequence of rational numbers $p_{n} / q_{n}=\left[a_{1}, a_{2}, \ldots, a_{n}\right]$ converges to $\alpha$. The irrational number $\alpha$ is a Diophantine number of order $\kappa \geq 2$ if there exists

Received November 17, 2009.

2010 Mathematics Subject Classification. Primary 37F50; Secondary 32A10, 37F99.

Key words and phrases. Siegel disks, wandering domains.

This work was supported by Grant-in-Aid for Young Scientists (B) No. 21740121 of Japan Society for the Promotion of Science. 
$\varepsilon>0$ such that

$$
\left|\alpha-\frac{p}{q}\right|>\frac{\varepsilon}{q^{\kappa}}
$$

for all rational numbers $p / q$. The irrational number $\alpha$ belongs to the class of Diophantine numbers of order $\kappa$ if and only if the sequence

$$
\left\{\frac{q_{n+1}}{q_{n}^{\kappa-1}}\right\}_{n=1}^{\infty}
$$

is bounded. In the case that $\kappa=2$, the sequence $\left\{a_{n}\right\}_{n=1}^{\infty}$ is bounded if and only if $\left\{q_{n+1} / q_{n}\right\}_{n=1}^{\infty}$ is. Therefore Diophantine numbers of order 2 are said to be of bounded type. The irrational number $\alpha$ is a Bryuno number if the sum

$$
\sum_{n=1}^{\infty} \frac{\log q_{n+1}}{q_{n}}
$$

converges. Bryuno proved that if $\alpha$ is a Bryuno number, then $f$ is locally linearizable at $z_{0}$. Yoccoz proved that if a quadratic polynomial $Q(z)=e^{2 \pi i \alpha} z+z^{2}$ is locally linearizable at the origin, then $\alpha$ is a Bryuno number.

In this paper, we consider transcendental entire functions

$$
f_{c}(z)=c\left(\frac{z}{c}\right)^{n} \exp \left\{\frac{1}{c}(\lambda-n)(z-c)\right\},
$$

where $n \geq 2, c \in \mathbb{C} \backslash\{0\}, \lambda=e^{2 \pi i \alpha}$ and $0<\alpha<1$ is a Bryuno number. Let $f=f_{1}$. The origin is a superattracting fixed point and $c$ is the center of a Siegel disk $\Delta$ with rotation number $\alpha$. The function $f_{c}$ has the following properties:

- The origin is a superattracting fixed point with multiplicity $n$.

- $c$ is a fixed point with multiplier $f_{c}^{\prime}(c)=\lambda$.

- $c n /(n-\lambda)$ is a critical point and there is no other critical point.

- The origin is an asymptotic value.

- There is no singular value other than the origin and $f_{c}(c n /(n-\lambda))$.

- $f_{c}$ is of finite order.

Main Theorem. If $n$ is large enough, then there exist bounded simply connected domains $U$ and $V$ satisfying $1 \in U \Subset V$ such that $f: U \rightarrow V$ is a quadratic-like map.

A map $g: U_{1} \rightarrow U_{2}$ is a polynomial-like map of degree $d$ if $g$ is a holomorphic proper map of degree $d$, where $U_{1}$ and $U_{2}$ are topological disks with $U_{1} \Subset U_{2}$. In the case that $d=2$, we call it a quadratic-like map. The filled-in Julia set $K(g)$ of the polynomial-like map $g: U_{1} \rightarrow U_{2}$ is defined as

$$
K(g)=\left\{z \in U_{1}: g^{n}(z) \in U_{1} \text { for all } n \geq 0\right\}
$$

and the Julia set $J(g)$ of the polynomial-like map is the boundary $J(g)=\partial K(g)$ of the filled-in Julia set. The dynamics and the structure of the Julia set of a polynomial-like map correspond to those of some genuine polynomial at the 


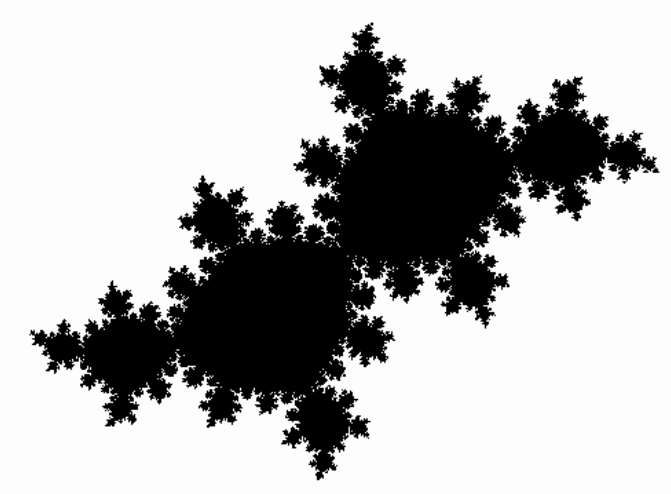

FiguRE 1. The Siegel disk of the quadratic polynomial $Q(z)=$ $e^{2 \pi i \alpha} z+z^{2}$ centered at the origin, where $\alpha=(\sqrt{5}-1) / 2=$ $[1,1,1, \ldots]$.

level of quasiconformality (The Straightening Theorem). Therefore properties of the Siegel disk $\Delta$ of $f$ centered at 1 and its boundary $\partial \Delta$ correspond to those of the Siegel disk $\mathcal{D}$ of $Q$ centered at the origin and its boundary $\partial \mathcal{D}$. For examples:

1. The function $f$ is locally linearizable at 1 if and only if $\alpha$ is a Bryuno number.

2. If $\alpha$ is of bounded type, then the boundary $\partial \Delta$ is a quasicircle containing the critical point $n /(n-\lambda)$.

3. The boundary $\partial \Delta$ is a quasicircle but the critical point $n /(n-\lambda)$ is not on $\partial \Delta$ for some $\alpha$.

Topological and quasiconformal statements which hold for the Siegel disk of the quadratic polynomial $Q$ centered at the origin hold for the Siegel disk of the transcendental entire function $f$ centered at 1 .

\section{Siegel disks of quadratic polynomials and quadratic-like maps}

Let $Q(z)=e^{2 \pi i \alpha} z+z^{2}$, where $0<\alpha<1$ is irrational. The quadratic polynomial $Q$ has the following properties:

- The point at infinity is a superattracting fixed point.

- The origin is a fixed point with multiplier $Q^{\prime}(0)=e^{2 \pi i \alpha}$.

- $-e^{2 \pi i \alpha} / 2$ is a critical point. 
If the irrational number $\alpha$ is a Bryuno number, then there exists the Siegel disk $\mathcal{D}$ of $Q$ centered at the origin. Many mathematicians have been interested in the boundary $\partial \mathcal{D}$ of the Siegel disk $\mathcal{D}$ for years and have obtained many results about it. A basic result about it is that the boundary $\partial \mathcal{D}$ is contained in the postcritical set of $Q$, namely

$$
\partial \mathcal{D} \subset \overline{\left\{Q^{n}\left(-e^{2 \pi i \alpha} / 2\right)\right\}_{n=1}^{\infty}} .
$$

The critical point belongs to the Julia set $J(Q)$. However we don't know whether it is on the boundary $\partial \mathcal{D}$ or not in general. Herman proved that if the irrational number $\alpha$ is of Herman-Yoccoz type (every analytic circle diffeomorphism with rotation number $\alpha$ is analytically linearizable), then the critical point is on the boundary $\partial \mathcal{D}$. The Siegel disk $\mathcal{D}$ is a simply connected domain. Hence there exists a conformal map between the Siegel disk and the unit disk. However we don't know whether the boundary $\partial \mathcal{D}$ is a closed Jordan curve or not in general. Many mathematicians contributed to the result that if the irrational number $\alpha$ is of bounded type, then the boundary $\partial \mathcal{D}$ is a quasicircle containing the critical point. Petersen and Zakeri proved that if the irrational number $\alpha$ is of David type (the irrational number $\alpha=\left[a_{1}, a_{2}, \ldots, a_{n}, \ldots\right]$ satisfies that $\log a_{n}=O(\sqrt{n})$ as $n$ tends to $\left.\infty\right)$, then the boundary $\partial \mathcal{D}$ is a David circle containing the critical point. David circles are closed Jordan curves. Moreover there exists an irrational number $\alpha$ of David type for which the boundary $\partial \mathcal{D}$ is a David circle but not a quasicircle. Therefore the above David-type result contains the bounded-type one. Herman proved that there exists a Bryuno number $\alpha$ such that the boundary $\partial \mathcal{D}$ is quasicircle but the critical point is not on $\partial \mathcal{D}$. Figure 1 is the Siegel disk $\mathcal{D}$, where the rotation number $\alpha=(\sqrt{5}-1) / 2=[1,1,1, \ldots]$ is of bounded type. Hence the boundary $\partial \mathcal{D}$ is a quasicircle containing the critical point.

\section{Polynomial-like maps}

A map $g: U_{1} \rightarrow U_{2}$ is a polynomial-like map of degree $d$ if $g$ is a holomorphic proper map of degree $d$, where $U_{1}$ and $U_{2}$ are topological disks with $U_{1} \Subset U_{2}$. In the case that $d=2$, we call it a quadratic-like map. The filled-in Julia set $K(g)$ of the polynomial-like map $g: U_{1} \rightarrow U_{2}$ is defined as

$$
K(g)=\left\{z \in U_{1}: g^{n}(z) \in U_{1} \text { for all } n \geq 0\right\}
$$

and the Julia set $J(g)$ of the polynomial-like map is the boundary $J(g)=\partial K(g)$ of the filled-in Julia set. Two polynomial-like maps $g$ and $h$ are hybrid equivalent if there exists a quasiconformal map $\varphi$ defined on a neighborhood of $K(g)$ such that $\bar{\partial} \varphi=0$ on $K(g)$ and $\varphi \circ g=h \circ \varphi$. The following theorem is called The Straightening Theorem. It means that the dynamics and the structure of the Julia set of a polynomial-like map correspond to those of some genuine polynomial at the level of quasiconformality. 
Theorem 2.1 (The Straightening Theorem). Every polynomial-like map $g$ is hybrid equivalent to some genuine polynomial $p$ of the same degree. Moreover if $K(g)$ is connected, then the polynomial $p$ is unique up to affine conjugation.

The Straightening Theorem indicates that topological and quasiconformal statements which hold for the Siegel disk $\mathcal{D}$ of the quadratic polynomial $Q$ hold for Siegel disks of quadratic-like maps.

\section{Siegel disks of transcendental entire functions}

\subsection{Preliminaries}

Let $f: \mathbb{C} \rightarrow \mathbb{C}$ be an entire function. The function $f$ is a transcendental entire function if $f$ does not extend to the point at infinity. In this case, the point at infinity is an essential singularity. A point $v \in \mathbb{C}$ is a critical value of $f$ if $v=f(c)$, where $c$ is a critical point of $f$. A point $v \in \mathbb{C}$ is an asymptotic value of $f$ if there exists a path $\gamma:[0,1) \rightarrow \mathbb{C}$ such that $\gamma(t) \rightarrow \infty$ and $f(\gamma(t)) \rightarrow v$ as $t \rightarrow 1$. The function $f$ is said to be of finite order if there exist positive constants $a$ and $r$ such that

$$
|f(z)| \leq \exp |z|^{a}
$$

for $|z|>r$. The infimum of all positive numbers $a$ for which the inequality holds is the order of $f$. Let $\left\{a_{j}\right\}$ be the zeros of $f$ listed with multiplicities. The rank $p$ of $f$ is the least positive integer such that

$$
\sum_{a_{j} \neq 0}\left|a_{j}\right|^{-(p+1)}<\infty
$$

If $f$ has a finite number of zeros, then it has rank zero. Suppose that $f$ is of finite order $\lambda$. Then the canonical Weierastrass product

$$
f(z)=e^{g(z)} P(z)
$$

has the property that $g$ is a polynomial of degree $q \leq \lambda$. The genus $\mu$ of $f$ is the maximum of $p$ and $q$.

Theorem 3.1. An entire function of finite genus $\mu$ is also of finite order $\mu$ and $\lambda<\mu+1$.

Theorem 3.2 (The Hadamard Factorization Theorem). An entire function of finite order $\lambda$ is also of finite genus $\mu$ and $\mu \leq \lambda$.

\subsection{Siegel disks and dynamics}

Let $f(z)=p(z) \exp (q(z))$, where $p$ and $q$ are polynomials. Zakeri proved the following theorem about Siegel disks of transcendental entire functions.

Theorem 3.3 (Zakeri, [30]). Suppose that the origin is a fixed point of $f$ with multiplier $f^{\prime}(0)=e^{2 \pi i \alpha}$. If $\alpha$ is of bounded type, then the boundary of the Siegel disk centered at the origin is a quasicircle containing at least one critical point of $f$. 
Zakeri also proved useful theorems about the function $f$ in [30]. Suppose that the degree of the polynomial $q$ is at least one.

Theorem 3.4 (Zakeri, [30]). The function $f$ has a unique asymptotic value at the origin.

Theorem 3.5 (Zakeri, [30]). Suppose that $V$ is a simply connected domain in $\mathbb{C} \backslash\{0\}$ and $U$ is a connected component of $f^{-1}(V)$. Then $f: U \rightarrow V$ is a proper map.

Hereafter we consider transcendental entire functions

$$
f_{c}(z)=c\left(\frac{z}{c}\right)^{n} \exp \left\{\frac{1}{c}(\lambda-n)(z-c)\right\}
$$

where $n \geq 2, c \in \mathbb{C} \backslash\{0\}, \lambda=e^{2 \pi i \alpha}$ and $0<\alpha<1$ is a Bryuno number. Let $f=f_{1}$. The origin is a superattracting fixed point and $c$ is the center of a Siegel disk $\Delta$ with rotation number $\alpha$. The function $f_{c}$ has the following properties:

- The origin is a superattracting fixed point with multiplicity $n$.

- $c$ is a fixed point with multiplier $f_{c}^{\prime}(c)=\lambda$.

- $c n /(n-\lambda)$ is a critical point and there is no other critical point.

- The origin is an asymptotic value (Theorem 3.4).

- There is no singular value other than the origin and $f_{c}(c n /(n-\lambda))$.

- $f_{c}$ is of finite order.

Lemma 3.6. Two functions $f_{c_{1}}$ and $f_{c_{2}}$ are conjugate via a conformal map for all non-zero complex numbers $c_{1}$ and $c_{2}$.

Proof. It is clear that $\varphi \circ f_{c_{1}} \circ \varphi^{-1}=f_{c_{2}}$, where $\varphi(z)=\left(c_{2} / c_{1}\right) z$.

Let $D(\varepsilon, r)$ be the disk with center $(1+\varepsilon) c$ and radius $r$,

$$
D(\varepsilon, r)=\{z \in \mathbb{C}:|z-(1+\varepsilon) c|<r\} .
$$

Lemma 3.7. If $\left|c_{0}\right|$ is large enough, then the following three statements hold for a small positive number $\varepsilon_{0}$ and a large integer $n_{0}$ :

(1) The inequality $\left|f_{c_{0}}(z)-c_{0}\right|>3 r$ holds on the circle $\partial D\left(\varepsilon_{0}, r\right)$.

(2) The inequality $r+\varepsilon_{0}\left|c_{0}\right|<2 r$ holds.

(3) The critical value $f_{c_{0}}\left(c_{0} n_{0} /\left(n_{0}-\lambda\right)\right)$ is in the disk $D(0,2 r)$.

Proof. Let $\varepsilon>0$ be small enough and $n \geq 2$ large enough such that $\Phi(\varepsilon, n)$ is near 1 , where

$$
\Phi(\varepsilon, n)=\left|e^{\lambda \varepsilon}\left(\frac{1+\varepsilon}{e^{\varepsilon}}\right)^{n}-1\right| .
$$


DYNAMICS OF TRANSCENDENTAL ENTIRE FUNCTIONS WITH SIEGEL DISKS 719 For $z=(1+\varepsilon) c+r e^{i \theta}$, we obtain that

$$
\begin{aligned}
\left|f_{c}(z)-c\right| & =|c| \cdot\left|\left(\frac{z}{c}\right)^{n} \exp \left\{\frac{1}{c}(\lambda-n)(z-c)\right\}-1\right| \\
& =|c| \cdot\left|\left(1+\varepsilon+\frac{r e^{i \theta}}{c}\right)^{n} \exp \left\{(\lambda-n)\left(\varepsilon+\frac{r e^{i \theta}}{c}\right)\right\}-1\right| \\
& =|c| \cdot\left|\exp \left\{\lambda\left(\varepsilon+\frac{r e^{i \theta}}{c}\right)\right\} \cdot\left\{\frac{1+\varepsilon+\frac{r e^{i \theta}}{c}}{\exp \left(\varepsilon+\frac{r e^{i \theta}}{c}\right)}\right\}^{n}-1\right|
\end{aligned}
$$

If $\left|c_{0}\right|$ is large enough, then

$$
\left|f_{c_{0}}(z)-c_{0}\right|>\frac{1}{2} \Phi(n, \varepsilon) \cdot\left|c_{0}\right|>\frac{1}{3} \Phi(n, \varepsilon) \cdot\left|c_{0}\right|>3 r .
$$

If $\varepsilon$ is small enough, then the inequality $r+\varepsilon\left|c_{0}\right|<2 r$ holds. Since

$$
\lim _{\varepsilon \rightarrow 0} \Phi(\varepsilon, n)=0, \quad \lim _{n \rightarrow \infty} \Phi(\varepsilon, n)=1
$$

and

$$
\begin{aligned}
& \lim _{n \rightarrow \infty} f_{c_{0}}\left(\frac{c_{0} n}{n-\lambda}\right) \\
= & \lim _{n \rightarrow \infty} c_{0}\left(\frac{n}{n-\lambda}\right)^{n} e^{-\lambda} \\
= & \lim _{n \rightarrow \infty} c_{0}\left(\frac{n}{n-\lambda}\right)^{\lambda}\left(\frac{n}{n-\lambda}\right)^{n-\lambda} e^{-\lambda} \\
= & \lim _{n \rightarrow \infty} c_{0}\left(\frac{1}{1-\lambda / n}\right)^{\lambda}\left\{\left(1+\frac{\lambda}{n-\lambda}\right)^{(n-\lambda) / \lambda}\right\}^{\lambda} e^{-\lambda} \\
= & c_{0} \cdot 1 \cdot e^{\lambda} \cdot e^{-\lambda}=c_{0},
\end{aligned}
$$

there exist a small positive number $\varepsilon_{0}$ and a large integer $n_{0}$ such that the inequality

$$
\left|f_{c_{0}}(z)-c_{0}\right|>\frac{1}{2} \Phi(\varepsilon, n) \cdot\left|c_{0}\right|>\frac{1}{3} \Phi\left(\varepsilon_{0}, n_{0}\right) \cdot\left|c_{0}\right|>3 r
$$

holds on the circle $\partial D\left(\varepsilon_{0}, r\right)$, the inequality $r+\varepsilon_{0}\left|c_{0}\right|<2 r$ holds and the critical value $f_{c_{0}}\left(c_{0} n_{0} /\left(n_{0}-\lambda\right)\right)$ is in the disk $D(0,2 r)$.

Remark 3.8. (i) The above statement (2) implies that the fixed point $c_{0}$ is in the disk $D\left(\varepsilon_{0}, r\right)$ and $D\left(\varepsilon_{0}, r\right) \Subset D(0,2 r)$.

(ii) The above three statements (1), (2) and (3) hold for all $n \geq n_{0}$. 

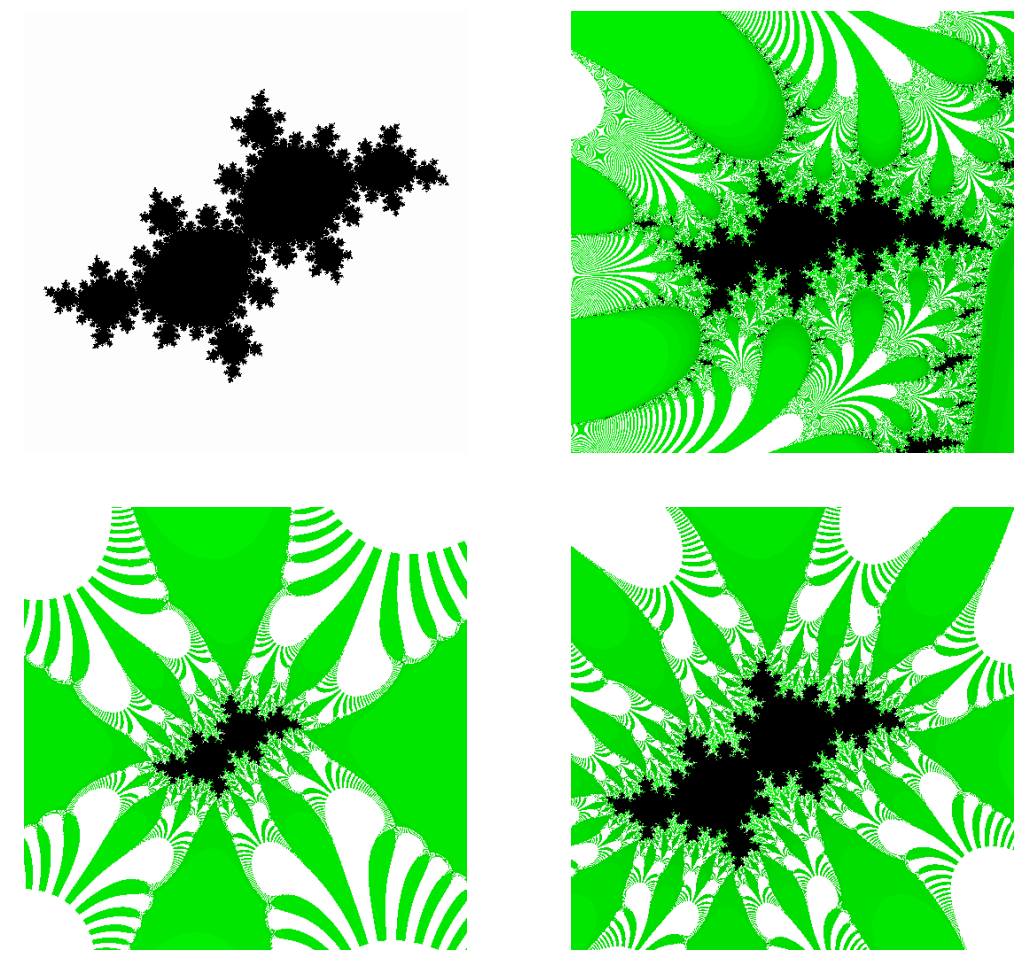

Figure 2. The upper left-hand figure is the Siegel disk $\mathcal{D}$ of the quadratic polynomial $Q(z)=e^{2 \pi i \alpha} z+z^{2}$ centered at the origin, where the rotation number $\alpha=(\sqrt{5}-1) / 2=$ $[1,1,1, \ldots]$. Another all figures are the Siegel disk $\Delta$ of the transcendental entire function $f$ centered at 1 . Black regions are the Siegel disk $\Delta$ and its preimages. Green ones are the attracting basin at the origin. The upper right-hand one is in the case that $n=2$. The lower left-hand one is in the case that $n=32$. It would be a "quadratic-polynomial-type" Siegel disk. The lower right-hand one is the enlargement of the lower left-hand one.

Theorem 3.9. Let $c_{0}, \varepsilon_{0}$ and $n_{0}$ be as in Lemma 3.7. Suppose that $n \geq n_{0}$ is large enough, $V=D(0,2 r)$ and $U$ is the connected component of $f_{c_{0}}^{-1}(V)$ containing the fixed point $c_{0}$. Then $f_{c_{0}}: U \rightarrow V$ is a quadratic-like map.

Proof. By Lemma 3.7, the inclusion $U \Subset V$ holds and the critical point $c_{0} n /(n-$ $\lambda)$ is in $U$. By Theorem 3.5, the restriction $f_{c_{0}}: U \rightarrow V$ is a proper map. Therefore $f_{c_{0}}: U \rightarrow V$ is a quadratic-like map. 
By Lemma 3.6, we obtain the Main Theorem:

Main Theorem. If $n$ is large enough, then there exist bounded simply connected domains $U$ and $V$ satisfying $1 \in U \Subset V$ such that $f: U \rightarrow V$ is a quadratic-like map.

\subsection{Corollaries}

Suppose that $n$ is large enough. Let $U$ and $V$ be above. By the Main Theorem, the restriction $f: U \rightarrow V$ is a quadratic-like map. Hence the Siegel disk $\Delta$ of $f$ centered at 1 is in $U$ and the shape looks like the Siegel disk $\mathcal{D}$ of the quadratic polynomial $Q$. Therefore we call Siegel disks of quadratic-like maps "quadratic-polynomial-type" Siegel disks. The Straightening Theorem indicates that topological and quasiconformal statements which hold for the Siegel disk $\mathcal{D}$ hold for the Siegel disk $\Delta$. For examples:

Corollary 3.10. The function $f$ is locally linearizable at 1 if and only if the rotation number $\alpha$ is a Bryuno number.

Corollary 3.11. If the rotation number $\alpha$ is of bounded type, then the boundary $\partial \Delta$ is a quasicircle containing the critical point $n /(n-\lambda)$.

Corollary 3.12. The boundary $\partial \Delta$ is a quasicircle but the critical point $n /(n-$ $\lambda$ ) is not on $\partial \Delta$ for some $\alpha$.

\section{Applications}

Definition 4.1. Let $g$ be a transcendental entire function and $U$ a Fatou component. The Fatou component $U$ is a wandering domain if all $g^{m}(U)$ with $m \geq 1$ are contained different Fatou components. The Fatou component $U$ is a Baker domain of period $p$ if $U$ is a periodic Fatou component of period $p$ such that the point at infinity is on the boundary $\partial U$ and $g^{p m}(z) \rightarrow \infty$ for all $z \in U$ as $m \rightarrow \infty$.

Let $0<\alpha<1$ be a Bryuno number. We consider the logarithmic lift of $f$,

$$
\tilde{f}(z)=n z+(\lambda-n)\left(e^{z}-1\right) .
$$

Then the functional equation $\exp \circ \tilde{f}=f \circ \exp$ holds. The Fatou component $B=\tilde{f}(B)$ such that $\exp B$ is the immediate basin of $f$ at the origin is an invariant Baker domain of $\tilde{f}$. The origin is a fixed point of $\tilde{f}$ with multiplier $\lambda=e^{2 \pi i \alpha}$. Let $\tilde{\Delta}$ be the Siegel disk of $\tilde{f}$ centered at the origin and $\tilde{\Delta}_{k}$ the Fatou component containing $2 \pi k i$, where $k$ is a non-zero integer. Since the functional equation exp $\circ \tilde{f}=f \circ \exp$ holds, the exponential map projects $\tilde{\Delta}$ and $\tilde{\Delta}_{k}$ down to the Siegel disk $\Delta$ of $f$ centered at 1 . The behavior of $2 \pi k i$ is

$$
2 \pi k i \stackrel{\tilde{f}}{\mapsto} 2 \pi k n i \stackrel{\tilde{f}}{\mapsto} 2 \pi k n^{2} i \stackrel{\tilde{f}}{\mapsto} \ldots \stackrel{\tilde{f}}{\mapsto} 2 \pi k n^{m} i \stackrel{\tilde{f}}{\mapsto} \cdots
$$

or $\tilde{f}^{m}(2 \pi k i)=2 \pi k n^{m} i$. Therefore $\left\{\tilde{\Delta}_{ \pm k}\right\}_{k}$ : a prime number is a family of infinitely many wandering domains having distinct orbits. 


\section{Summary}

The logarithmic lift $\tilde{f}$ has the following properties:

- The Fatou component $B=\tilde{f}(B)$ such that $\exp B$ is the immediate basin of $f$ at the origin is an invariant Baker domain.

- The Siegel disk $\tilde{\Delta}$ of $\tilde{f}$ centered at the origin projects down to the Siegel disk $\Delta$ of $f$ centered at 1 via the exponential map.

- Any Fatou component $\tilde{\Delta}_{k}$ containing $2 \pi k i$ is a wandering domain and projects down to the Siegel disk $\Delta$ of $f$ centered at 1 via the exponential map.

- $\left\{\tilde{\Delta}_{ \pm k}\right\}_{k}$ : a prime number is a family of infinitely many wandering domains having distinct orbits.

- $\omega_{k}=\log |n /(n-\lambda)|+i \cdot[\arg \{n /(n-\lambda)\}+2 \pi k]$ are critical points.

The following is an application of the Main Theorem:

Corollary 4.2. If $n$ is large enough, then the following statements hold:

(1) The Siegel disk $\tilde{\Delta}$ of $\tilde{f}$ centered at the origin is a "quadratic-polynomial-type" Siegel disk.

(2) The shape of Wandering domains $\tilde{\Delta}_{k}$ containing $2 \pi k i$ looks like the Siegel disk $\Delta$ of $f$ centered at 1.

Corollary 4.2 indicates that if the irrational number $\alpha$ is of bounded type, then

- the boundary $\partial \tilde{\Delta}$ is a quasicircle containing the critical point $\omega_{0}$,

- the boundary $\partial \tilde{\Delta}_{k}$ is also a quasicircle containing the critical point $\omega_{k}$.
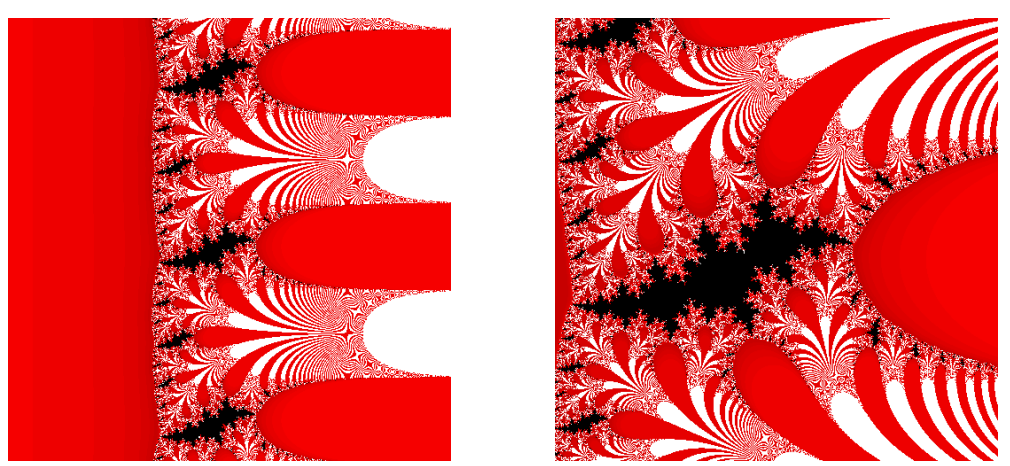

Figure 3 . The two figures are in the case that $n=2$ and the rotation number $\alpha=(\sqrt{5}-1) / 2=[1,1,1, \ldots]$. The left-hand one is the Siegel disk $\tilde{\Delta}$ centered at the origin and wandering domains $\tilde{\Delta}_{1}$ and $\tilde{\Delta}_{-1}$. The right-hand one is the enlargement of the left-hand one. Black regions are the Siegel disk $\Delta$ or wandering domains $\tilde{\Delta}_{k}$ and their preimages. Red ones are the Baker domain $B$ and its preimages. 


\section{References}

[1] L. V. Ahlfors, Lectures on Quasiconformal Mappings, Second edition, With supplemental chapters by C. J. Earle, I. Kra, M. Shishikura and J. H. Hubbard. University Lecture Series, 38. American Mathematical Society, Providence, RI, 2006.

[2] A. Avila, X. Buff and A. Chéritat, Siegel disks with smooth boundaries, Acta Math. 193 (2004), no. 1, 1-30.

[3] A. F. Beardon, Iteration of Rational Functions, Complex analytic dynamical systems, Graduate Texts in Mathematics, 132. Springer-Verlag, New York, 1991.

[4] R. Berenguel and N. Fagella, An entire transcendental family with a peersistent Siegel disc, http://www.gsd.uab.es/personal/nfagella.

[5] P. Blanchard, Complex analytic dynamics on the Riemann sphere, Bull. Amer. Math. Soc. (N.S.) 11 (1984), no. 1, 85-141.

[6] X. Buff and A. Chéritat, Quadratic Julia sets with positive area, http://www.picard.upstlse.fr/ buff/Preprints/Preprints.html.

[7] X. Buff and C. Henriksen, Scaling ratios and triangles in Siegel disks, Math. Res. Lett. 6 (1999), no. 3-4, 293-305.

[8] L. Carleson and T. Gamelin, Complex Dynamics, Springer-Verlag, 1993.

[9] A. Douady and J. H. Hubbard, On the dynamics of polynomial-like mappings, Ann. Sci. École Norm. Sup. (4) 18 (1985), no. 2, 287-343.

[10] N. Fagella and L. Geyer, Surgery on Herman rings of the complex standard family, Ergodic Theory Dynam. Systems 23 (2003), no. 2, 493-508.

[11] N. Fagella and C. Henriksen, The Teichmüller space of an entire function, Complex dynamics, 297-330, A K Peters, Wellesley, MA, 2009.

[12] A. Fletcher and V. Markovic, Quasiconformal Maps and Teichmüller Theory, Oxford Graduate Texts in Mathematics 11, Oxford University Press, Oxford, 2007.

[13] L. Geyer, Siegel discs, Herman rings and the Arnold family, Trans. Amer. Math. Soc. 353 (2001), no. 9, 3661-3683.

[14] J. Graczyk and P. Jones, Dimension of the boundary of quasiconformal Siegel disks, Invent. Math. 148 (2002), no. 3, 465-493.

[15] K. Katagata, Some cubic Blaschke products and quadratic rational functions with Siegel disks, Int. J. Contemp. Math. Sci. 2 (2007), no. 30, 1455-1470.

[16] _ Dynamics of rational functions and rational semigroups on the Riemann sphere, Thesis, Shimane University, 2008.

[17] _ Blaschke products and rational functions with Siegel disks, J. Korean Math. Soc. 46 (2009), no. 1, 151-170.

[18] - Dynamics of rational functions with Siegel disks and polynomial semigroups, Mem. Fac. Sci. Eng. Shimane Univ. Ser. B Math. Sci. 42 (2009), 17-57.

[19] A. Katok and B. Hasselblatt, Introduction to the Modern Theory of Dynamical Systems, Encyclopedia of Mathematics and its Applications 54, Cambridge University Press, 1995.

[20] K. Linda and Z. Gaofei, Bounded type Siegel disks of a one dimensional family of entire functions, preprint.

[21] C. T. McMullen, Self-similarity of Siegel disks and Hausdorff dimension of Julia sets, Acta Math. 180 (1998), no. 2, 247-292.

[22] W. de Melo and S. van Strien, One-dimensional Dynamics, Ergebnisse der Mathematik und ihrer Grenzgebiete (3) 25, Springer-Verlag, Berlin, 1993.

[23] J. Milnor, Dynamics in One Complex Variable, Vieweg, 2nd edition, 2000.

[24] S. Morosawa, Y. Nishimura, M. Taniguchi, and T. Ueda, Holomorphic Dynamics, Cambridge Studies in Advanced Mathematics 66, 2000.

[25] C. L. Petersen, On holomorphic critical quasi-circle maps, Ergodic Theory Dynam. Systems 24 (2004), no. 5, 1739-1751. 
[26] N. Steinmetz, Rational Iteration, Complex analytic dynamical systems, de Gruyter Studies in Mathematics, 16, Walter de Gruyter \& Co., Berlin, 1993.

[27] M. Yampolsky and S. Zakeri, Mating Siegel quadratic polynomials, J. Amer. Math. Soc. 14 (2001), no. 1, 25-78

[28] S. Zakeri, Old and new on quadratic Siegel disks, http://www.math.qc.edu/ zakeri/ papers/papers.

[29] , Dynamics of cubic Siegel polynomials, Comm. Math. Phys. 206 (1999), no. 1, $185-233$

[30] - On Siegel Disks of a Class of Entire Maps, http://www.math.qc.edu/ zakeri/ papers/papers.html.

Ichinoseki National College of Technology

Takanashi, Hagisho, Ichinoseki, Iwate 021-8511, Japan

E-mail address: katagata@ichinoseki.ac.jp 